\title{
Characterization of FDTD artifacts and modes in photonic crystals
}

\author{
José M. López-Alonso, José M. Rico-García, Javier Alda \\ Optics Department, University Complutense of Madrid, \\ School of Optics. Avda. Arcos del Jalón s/n, 28037 Madrid, Spain \\ Phone: +34 9139468 72, Fax: +34 913946885 \\ E-mail: jmrico@fis.ucm.es jmlopez@opt.ucm.es, j.alda@fis.ucm.es
}

\begin{abstract}
FDTD algorithms are being used as a numeric tool for the analysis of photonic crystals. The definition of the modes associated with them is of interest for the study of the capabilities of photonic crystal devices. The Principal Component Analysis (PCA) has been applied here to a sequence of images corresponding to the electromagnetic fields obtained from the FDTD simulations. PCA has revealed and quantified the importance of the modes appearing in the photonic crystals. The capability of PCA to produce spatial structures, or maps, associated with temporal evolutions has made possible the calculation of the modulus and phase of the modes existing in the photonic crystal. Some other modes, contributing with an almost negligible amount to the total variance of the original data, are also revealed by the method. Besides, PCA has been used to quantify the contribution of the numerical noise of the algorithm and to identify the effect of artifacts related with the matching of the computational grid and the inner geometry of the photonic crystal.
\end{abstract}

\section{Introduction.}

Photonic crystals are being currently considered as a major point of interest for researching in optics. The amount of practical devices incorporating photonic crystal is increased as the fabrication capabilities are improved and the modeling and theoretical analysis of these structures are more complete and accurate ${ }^{1,2,3}$. The Finite-Differences in the Time-Domain (FDTD) method is one of the numerical tools of computational electromagnetism used to evaluate the behavior of photonic crystal $^{4,5}$. FDTD serves as a numerical touchstone that validates designs and conceptions. At the same time FDTD has showed several limitations. Some of them are intrinsic and are very much unavoidable. Some others can be overcome by customized techniques or by a dedicated post-processing of the data obtained from the algorithm. In this contribution we show how a multivariate statistical technique may find application in the treatment and interpretation of the FDTD outputs. Principal Component Analysis (PCA) has been successfully applied to the characterization of covariance structures in several fields ranging from the analysis of noise in a sequence of frames to the identification of rare events in the stock market ${ }^{6,7,8}$. In the case treated here, the FDTD results are taken as a collection of frames equally spaced in time. Then, PCA is applied to them in order to find spatial-temporal structures with a clear physical meaning. For photonic crystal simulation using FDTD techniques, PCA has been used to filter numerical noise artifacts and to identify spatial temporal patterns corresponding with modes and field structures.

Section 2 of this paper makes a brief introduction of the PCA method. We emphasize in the parameters better adapted to the analysis of the results. As far as we are not interested in the fundamentals of FDTD, we directly treat with the specification of the practical case of the 
photonic crystals analyzed here ${ }^{5}$. The output of the FDTD method is prepared, analyzed and interpreted in Section 3 under the application of the PCA technique. Finally, section 4 summarizes the main results of the paper.

\section{Fundamentals of the Principal Component Analysis applied to Photonic Crystal Characterization by FDTD.}

PCA is based on multivariate statistical techniques. It has found application in a wide variety of fields. For the case treated here we consider the input data as a collection or sequence of frames, $\left\{F_{1}, F_{2}, \ldots, F_{i}, \ldots, F_{N}\right\}$, equally spaced in time, containing the 2-dimensional field distributions obtained from FDTD calculations, $F_{i}$. Then, the type of analysis applicable here will be quite similar to that one used for the characterization of noise in a sequence of infrared images. The frames contain a large number of pixels, $M$, that are considered, within the multivariate statistical approach, as $M$ realizations of a $N$-dimensional variable, being $N$ the number of frames included in the data set. The result of the blind application of the PCA technique is a collection of a modified set of $N$ principal components, $\left\{Y_{1}, Y_{2}, \ldots, Y_{\alpha}, \ldots, Y_{N}\right\}, N$ eigenvectors, $\lambda_{\alpha}$, and $N$ eigenvalues, $e_{\alpha}$. The $\mathrm{N}$ principal components obtained here can be interpreted as 2dimensional field distributions that explain the variance of the original data in decreasing order. The relation between the original data set and the principal component set is given by:

$$
Y_{\alpha}=\sum_{t_{i}=1}^{N} e_{\alpha}\left(t_{i}\right) F\left(t_{i}\right) \text {, }
$$

where $t_{i}$ means the time instant when the frame is taken. The value of the variance associated with each principal component, $Y_{\alpha}$, is given by the corresponding eigenvalue, $\lambda_{\alpha}$. Besides, the temporal evolution of the principal component is described by the associated eigenvector, $e_{\alpha}$. Both the eigenvalues and the eigenvectors are related by the following eigenvalue equation:

$$
S e_{\alpha}=\lambda_{\alpha} e_{\alpha},
$$

where $\mathrm{S}$ is the covariance matrix among frames. A graphical scheme of the PCA method is outlined in Figure 1.

Until here, PCA can be seen as a mere transformation of coordinates that can be explained as a rigid rotation from the $\mathrm{N}$-dimensional frame space to the $\mathrm{N}$-dimensional principal component space. Fortunately, we developed a sound method to automatically reduce the amount of significant principal component ${ }^{6}$. The classification of the principal component is made by grouping them into spatial temporal processes. Successive principal components will be grouped together when the uncertainties of the associated eigenvalues are consecutively undistinguishable. The application of this classification strategy makes possible to focus our attention in a very short number of processes, instead of analyzing a set of principal components having the same dimension than the original data set. Therefore, the main advantage of this approach is the reduction in the complexity of the data. Typically, a large subset of principal components associated with values of a ranging from a moderate value to the last one, $\alpha=N$, are grouped together and account for a variety of noise sources that most of the time are undistinguishable. The addition of the eigenvalues forming part of a given process, $P_{j}$, describes the contribution of this process to the total variance of the data. This can be seen as a parameter quantifying the noise and it is defined in the following way, 


$$
\Omega_{P_{j}}=\frac{\sum_{\alpha \in P_{j}} \lambda_{\alpha}}{\sum_{\alpha=1}^{N} \lambda_{\alpha}},
$$

where $P_{j}$ means the process containing a finite subset of eigenvectors, eigenvalues and principal components.

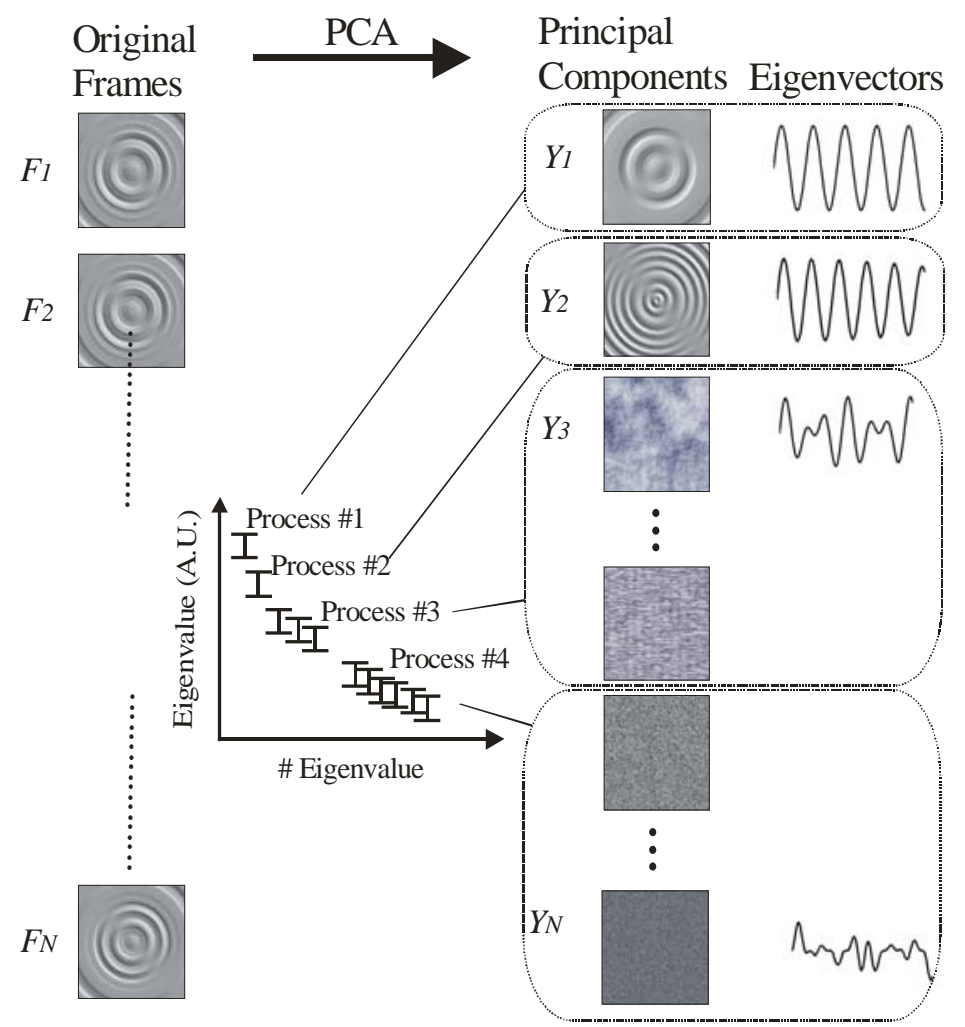

Figure 1: The PCA transform the original data set into a collection of eigenimages (the principal components) that are grouped by using the uncertainties of their eigenvalues. At the same time, the temporal evolution of each principal component is given by its associated eigenvector.

On the other hand, the first principal components or processes, containing very useful information related with the field distributions obtained from the FDTD. An added advantage of the PCA method is that the splitting of the data in orthogonal unconnected structures of the covariance makes possible the filtering of the data. Then, it is possible to retrieve a filtered version of the original data by taking into account only those principal components or processes with a given significance or interest. This is possible by using the following relation:

$$
F_{\left\{P_{j}, \ldots, P_{k}\right\}}\left(t_{i}\right)=\sum_{\alpha \in\left\{P_{j}, \ldots, P_{k}\right\}} e_{\alpha}\left(t_{i}\right) Y_{\alpha},
$$

where the sum is only made for the index included in the selected subset of principal components and processes, $\left\{P_{j}, \ldots, P_{k}\right\}$. Besides, when the eigenvalue associated with a principal component cannot be connected with its neighbors, its eigenvector shows a harmonic dependence. We will name these processes as single-value processes, or "quasi-monochromatic" processes?. In this case, this harmonic dependence indicates the presence of a very well defined temporal 
frequency. This frequency is an important feature of the field distribution itself and can be interpreted as the frequency of the mode surviving within the photonic crystal. The importance of the mode within the structure is given by the corresponding eigenvalue. By using this property, the PCA is well adequate to study physical situations where harmonic time dependences exist.

The linear decomposition produced by the PCA (see equation (4)) is appropriate to obtain the modes as the harmonic components of the resulting principal components. These harmonic components were defined previously as the quasi-monochromatic processes.

\section{Mode Analysis and Artifacts PCA filtering in FDTD}

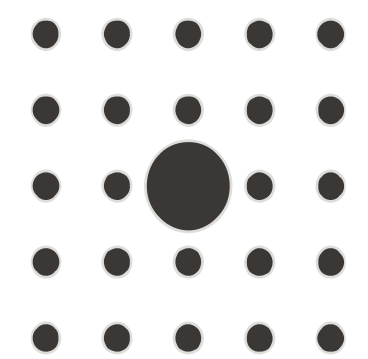

Figure 2: Physical arrangement of cylinders and central defect for the photonic crystal under analysis. The calculation grid for the FDTD evaluation is superimposed over this geometry. This plot represents the XY plane. The electric field vector is perpendicular to this plane for the analyzed cases.

\subsection{Parametrization of the photonic crystal and the FDTD calculations}

The type of photonic crystal studied by using the PCA method is an arrangement of 2dimensional cylinders equally spaced ${ }^{5}$ (see Figure 2). These cylinders are made of GaAs, having an index of refraction of 3.4 for the wavelength range studied here. The distance between adjacent center of cylinders is $1 \mu \mathrm{m}$. The modification of the bandgap of this structure is produced by the central cylinder. This cylinder has a diameter 3 times larger than the rest of the cylinders. The forbidden bandgap associated with this structure ranges from $0.87 \times 10^{14} \mathrm{~Hz}$ to $1.26 \times 10^{14} \mathrm{~Hz}$. The defect in the geometry makes possible a confinement of the electromagnetic field only for a selected set of frequencies within the bandgap: $0.891 \times 10^{14} \mathrm{~Hz}, 0.957 \times 10^{14} \mathrm{~Hz}, 1.0035 \times 10^{14} \mathrm{~Hz}$, and $1.1748 \times 10^{14} \mathrm{~Hz}$. These frequencies are those of the modes existing in the photonic crystal. In this case the photonic crystal behaves as a microcavity. In this point we should remark that although the analysis has been made for a very well defined system, the method of PCA filtering is applicable to any other structure.

After defining the photonic crystal, the FDTD grid is set for the evaluation of the electromagnetic field surviving in the structure ${ }^{9}$. The selected grid has a spatial period of $0.025 \mu \mathrm{m}$, and the Courant factor relating the spatial and temporal periods is 0.7068 . The simulations have been made by injecting a given excitation with the electric vector perpendicular to the plane of the arrangement. This is a TMz excitation (we are assuming that the $X Y$ plane is perpendicular to the axis of the cylinders). Depending on the location of the feeding point, some specific modes are selected because of the symmetry, and survive in the photonic crystal. 


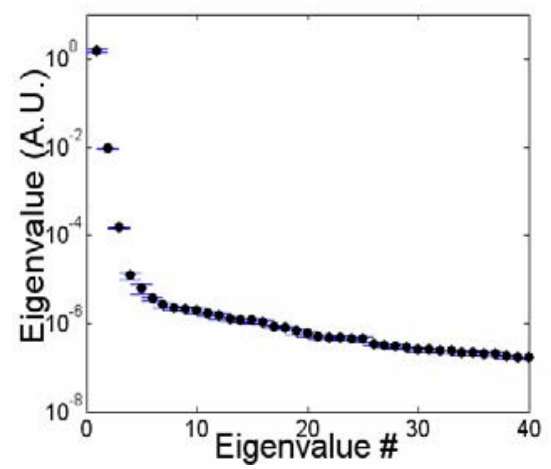

Figure 3: Values of the Eigenvalues, $\lambda_{\alpha}$, after applying the PCA method. Note the logarithm scale that points out the strong contribution of the very fist principal components. The uncertainties are also plotted. They serve to group the principal components into processes.

\subsection{Mode filtering by using PCA}

The results obtained from the FDTD calculation for a given excitation are arranged as a sequence of frames. The number of frames used in this study is $N=100$. Each frame corresponds with the spatial distribution of the $z$ component of the electric field, $E_{z}$ (we are assuming that $E_{x}=E_{y}=0$ ). The PCA method works blindly on the input data and produces a collection of parameters having very well defined meanings that deserves an adequate description. Firstly, the eigenvalues, $\lambda_{\alpha}(\alpha=1 \ldots 100)$, explain in decreasing order the importance of the corresponding principal components in the original data. This importance is defined as its contribution to the total variance of the original data. In order to illustrate the procedure we will make our discussion based on the results obtained for a centered excitation located at the center of the defect. This location also corresponds with the center of the computational grid. The mode surviving in the photonic crystal is the monopolar mode having a frequency of $1.0035 \times 10^{14} \mathrm{~Hz}$. Figure 3 shows the first 60 eigenvalues represented in a semilog plot.
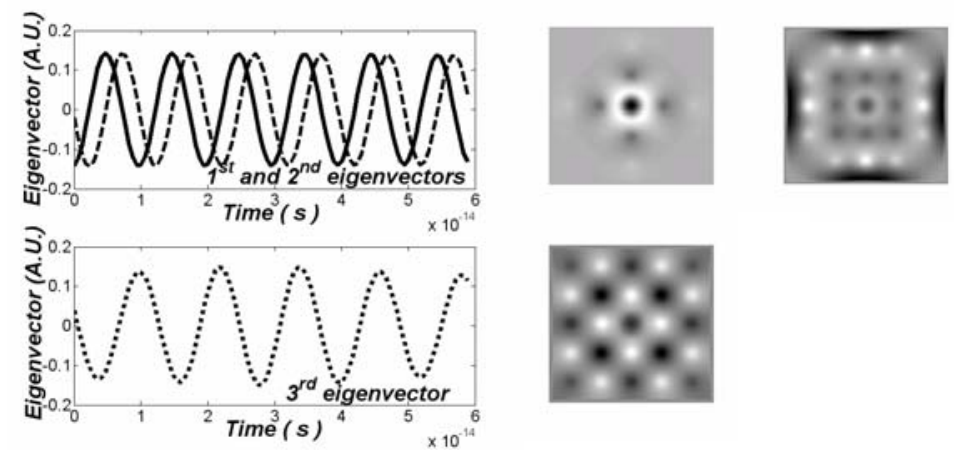

Figure 4: Plot of the eigenvectors associated with the first, second and third principal components. At the right we have plotted the spatial patterns corresponding to these principal components. The first two principal components, $Y_{1}, Y_{2}$, are single-value processes having the same frequency, but shifted $\pi / 2$ one with respect to the other. The third principal component, $Y_{3}$, resembles very much the inner geometry of the photonic crystal. All these results are obtained for a centered monopolar excitation.

For the photonic crystal analyzed here, the first principal component, $Y_{1}$, explains $99.39 \%$ of the total variance ${ }^{9}$. It constitutes a single-value process with a very well defined spatial distribution. This spatial distribution is plotted in Figure 4. When comparing this plot with the electric 
field distribution of the monopolar mode it is possible to clearly identify it as a PCA filtered version of this mode. The second principal component, $Y_{2}$, explains $0.60 \%$ of the total variance (two orders of magnitude below the first one). This principal component also defines a singlevalue process. However, it shows a very well defined spatial distribution that is presented also in Figure 4. Moreover, the temporal evolutions of these two principal components are harmonic functions having a temporal frequency equal to the expected frequency of the monopolar mode (see Figure 4). These harmonic functions are shifted by $\pi / 2$. This fact makes possible the interpretation of the second mode as the imaginary part of a complex process having a real part defined by the first principal component ${ }^{9}$. Then, it is possible to obtain the modulus and the phase of this complex process that should be interpreted as the modulus and the phase of the monopolar mode characteristic of the photonic crystal. The result obtained are presented in Figure 5.
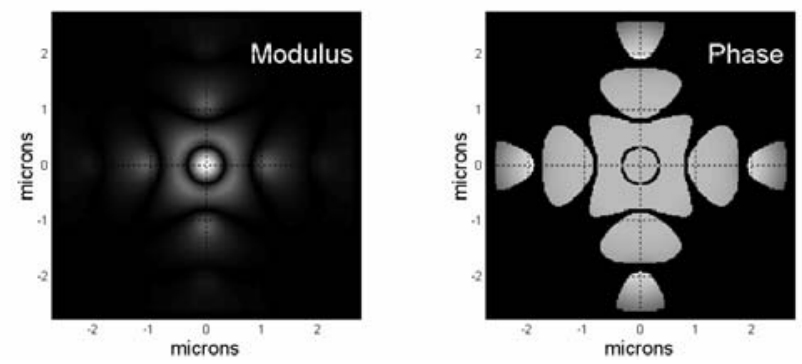

Figure 5: In the case of monopolar excitation, the first two principal components can be arranged together to produce a complex process. The modulus and the phase of this complex process can be easily obtained.

The third principal component, $Y_{3}$, also appears in the eigenvalue plot (figure 3 ) as a singlevalue process. Its temporal evolution and spatial distribution is also presented in Figure 4. The temporal frequency associated with this principal component is $0.843 \times 10^{14} \mathrm{~Hz}$, that lies outside the bandgap of the photonic crystal. In the case presented here, the temporal evolution of the electric field suggests the existence of a standing wave surviving within the structure. It should be noted that the relative importance of this process is $0.0096 \%$. However, the PCA method has been able to detect it and presents clearly its spatial and temporal distributions. When analyzing the energy distribution of this process we have found it very much associated with the existence of the cylinders. This behavior has been also observed for some other excitation arrangements.

This standingwave behavior corresponds with frequencies lying outside the bandgap. The explanation of this fact could be related with the method of generating the excitation in the FDTD scheme. The electric field source is a harmonic function fed at a given location depending on the desired excited mode symmetry, and it works for a limited period of time. Then the source is pulled out of the grid calculation and the electric field evolves within the structure. This finite period of time introduce a broadening of the spectrum of the excitation. This spectrum reaches the limits of the bandgap with a very small but non-zero power, allowing the excitation of frequencies outside the bandgap.

To better understand and justify this interpretation we have analyzed more in deep the behaviour of the quasi-monochromatic process for the hexapolar and monopolar excitations. The plot on the left of Figure 6 represents the electric field image corresponding with the standing wave process for a hexapolar mode excitation. The value of the temporal frequency associated with the standingwave process is centered around $1.6 \times 10^{14} \mathrm{~Hz}$. This frequency lies outside the bandgap limits. The associated wavelength within the material is equal to $\lambda_{m}=0.55 \mu \mathrm{m}$. The spatial 
pattern shown in the figure resembles the kind electric distribution expected for a resonance within the inner cylinder and having 5 periods. The radius of the circle having a length of 5 times the calculated wavelength is $0.44 \mathrm{~mm}$ (this circle has been plotted in the figure as a double dashed line). When the monopolar mode is excited within the microcavity it is also possible to calculate the absolute value of the instantaneous Poynting vector. A detail of this calculation is shown in the right part of Figure 6. The white circles represent the location and size of the small cylinders of the structure (the large black circle represents the central cylinder).

The energy seems to be coupled within the cylinders and showing a distribution within the cylinder with a central hole. To make a first order interpretation of this distribution we have calculated the size of the circle obtained when those rays inciding on the inner surface of the cylinder reflect on total reflection conditions and propagate bouncing within the cylinder. The radius of circle is given as $r=R / n$, where $R$ is the radius of the cylinder and $n$ is the index of refraction. When this calculation is made in the case treated here we obtain the circles represented in black. We can check that they fit very well with the size of the hole in the Poynting vector distribution of the standing wave mode.
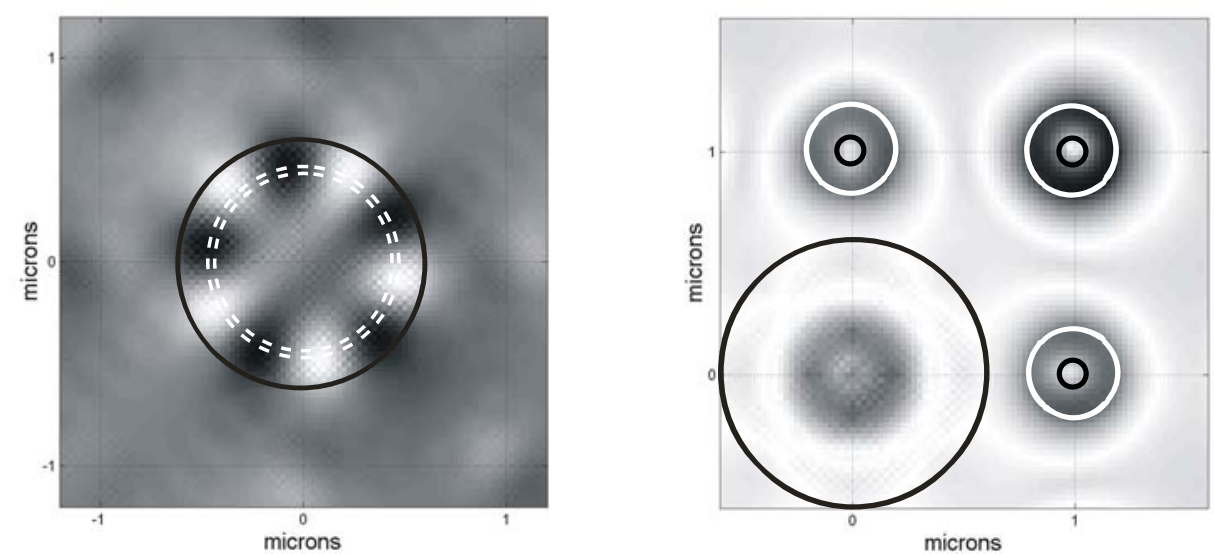

Figure 6: Standing wave behavior. Left: Central portion of the field distribution corresponding to the third principal component for a hexapolar mode excitation, the black circle represents the size of the central cylinder. Right: Detail of the absolute value of the instantaneous Poynting vector obtained for the third principal component for a monopolar mode excitation. The black circle represents the central cylinder, and the white circles correspond with the adjacent cylinders of the photonic crystal.

\subsection{Computational artifacts quantified and revealed by PCA}

FDTD is affected by computational artifact intrinsically. Some of these artifacts are due to numeric truncation, or to other specific problems of the machines and languages used to implement the algorithms. These are unavoidable. However PCA method is able to quantify them and filter them out from the resulting data ${ }^{10}$. For example, in the case analyzed in the previous section corresponding with the excitation of the monopolar mode in a photonic crystal we could identify a given portion of principal components, starting with the $6^{\text {th }}$ eigenvalue, grouped together in a noise process that carries out the $0.003 \%$ of the total variance. This amount of variance can be related with the numeric noise of the algorithm. 
Besides the numeric noise, some other artifacts arise when naïve errors are committed. One of them is related with the exact location of the excitation. In the previous discussion we did not pay too much attention to the adjustment of the computational grid and the symmetry of the photonic crystal under study. A closer look to this question reveals that an even number of points along one of the direction of the grid may not be able to locate precisely the center of the photonic crystal. In such a case, the excitation of the monopolar mode could be compromised. To prove that we have applied the PCA method to two sets of FDTD sequences having the same number of iterations, the same frequency of the excitation, but varying the number of points on the grid. One of them, that was labeled as the centered case had $221 \times 221$ points, and the other, the decentered case had $222 \times 222$. The spatial period remains the same for both simulations. However in the decentered case there were no grid points at the exact center of the photonic crystal. Actually, the excitation was located along one of the diagonals and displaced $0.0125 \mu \mathrm{m}$ from the center in each direction. After applying the PCA blind algorithm to the centered and decentered case we could find that the fourth principal component contributed almost three times more in the decentered case than in the centered one ${ }^{9,10}$. At the same time the first three principal components contributed almost the same for the two cases. When analyzing the spatial distribution of this fourth principal component we could check that the differences were distributed in a very different way (see figure 6). The fourth principal component had the same spatial distribution than the hexapolar mode of the photonic crystal. Moreover, the hexapolar mode needs the existence of an excitation along the diagonal of the rectangular arrangement. These two facts were clearly connected with the minimum displacement of the location of the excitation in the case of the decentered simulation. Then, we could check how the PCA method could reveal the existence of the hexapolar as an artifact related with a wrong election of the computation grid. It is interesting to note that the percentage of the variance of the original data related with this fourth principal component was only $0.0017 \%$. Such almost negligible contribution would be wiped out by a typical filtering process. However, the capability of PCA to show the spatial distribution of the variance contributions made easy the analysis of this case.
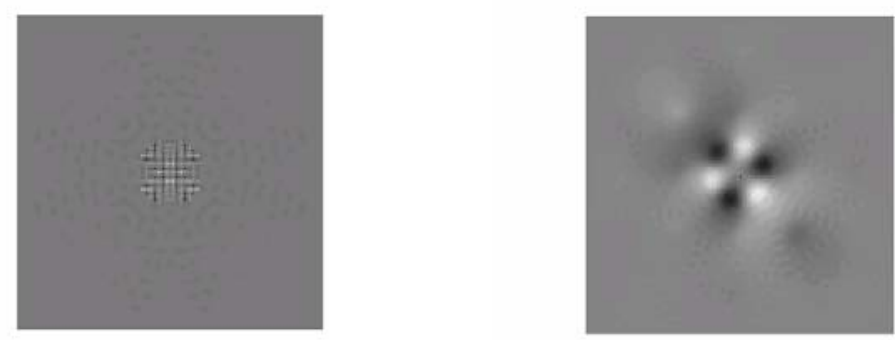

Figure 6: Spatial distribution of the fourth principal component for a exactly centered monopolar excitation (left) and for another excitation with a minimum amount of decentration (right)

\section{Conclusions}

The use of the PCA method to the FDTD results introduces some capabilities that could be of interest in the analysis of the spatial-temporal structures confined within photonic crystals. The automatic classification of the principal components into processes reduces the complexity of the analysis. It makes possible the filtering or rectification of the data based on the spatial and temporal evolution of the principal components. Some of the results can be interpreted in cou- 
ples to describe the modes both in modulus and phase. The existence of variance structures linked with the geometry of the photonic crystal, as the standing wave behavior, arise from the analysis. This is possible even when the contributions of these spatial-temporal patterns are very small. The classification of noise processes and its quantification define an uncertainty of the filtered values. These undesirable noise processes can be originated from the numeric errors, or even it could appear because of an unfortunate, although very small, selection of the computational grid.

We think that some other phenomena related with the presence and persistence of spatial temporal structures in the photonic crystal can be revealed by the PCA method. Its application is blind and automatic. The interpretation of the result is assisted by the capability of the PCA method to show the spatial and temporal evolution of the variance structures. Then, PCA becomes a useful tool for the analysis of field evolutions obtained from FDTD algorithms.

\section{Acknowledgments}

This research has been possible by the funding of the Ministerio de Ciencia y Tecnología of Spain through the project TIC2001-1259.

\section{References}

1. E. Yablonovitch, "Inhibited spontaneous emission in solid-state physics and electronics,” Phys. Rev. Lett. 58, 2059-2062 (1987).

2. J. D. Joannopoulos, R. D. Meade, J. N. Winn, Photonic Crystals, (Princeton University Press, New Jersey, 1995)

3. P. R. Villeneuve, S. Fan, and J. D. Joannopoulos, "Microcavities in photonic crystals: mode symmetry, tunability, and coupling efficiency,” Phys. Rev. B, 54, 7837-7842 (1996).

4. Taflove and S. C. Hagness Computacional Electrodynamics: The Finite-Difference Time Domain Method, 2nd ed. (Artech House, Boston, 2000).

5. S. Guo and S. Albin, "Numerical techniques for excitation and analysis of defect modes in photonic crystals," Opt. Express, 11, 1080-1089 (2003)

6. J. M. López-Alonso, J. Alda, and E. Bernabeu, "Principal component characterization of noise for infrared images,” Appl. Opt., 41, 320-331 (2002)

7. J. M. López-Alonso, J. Alda, "Operational parametrization of the $1 / \mathrm{f}$ noise of a sequence of frames by means of the principal components analysis in focal plane arrays," Opt. Eng., 42, 427-430 (2003)

8. J. M. López-Alonso, J. Alda, "Correlation in finance: a statistical approach”, Procc. SPIE, 5471, 311-321 (2004).

9. J. M. López-Alonso, J. M. Rico-García, J. Alda, "Photonic cristal characterization by FDTD and Principal Component Analysis”, Opt. Express, 12, 2176-2186 (2004)

10. J. M. López-Alonso, J. M. Rico-García, J. Alda, "Numerical artifacts in FDTD algorithms analyzed by means of Principal Components" submitted to IEEE Transaction on Antennas and Propagation (2004). 